\title{
Meta-análise do papel do exercício no tratamento da obstipação
}

\author{
Thomas Weiser, PhD, Sabine Landes, PhD
}

\begin{abstract}
As diretrizes atuais aconselham alterações no estilo de vida (incluindo mais exercício físico) como primeira medida para tratar a obstipação. Esta meta-análise investigou a influência da terapia de exercício físico nos sintomas de pacientes com obstipação crónica. Cerca de metade dos participantes num programa de exercício físico com a duração de várias semanas sentiram um efeito positivo nos seus sintomas. Estudos efetuados com os laxantes bisacodil e picossulfato de sódio demonstraram que estes, duma maneira geral, aliviaram os sintomas logo no primeiro dia de tratamento.
\end{abstract}

A obstipação é um problema de saúde que diminui de modo significativo a qualidade de vida das pessoas que dela sofrem [4] e apresenta consideráveis implicações económicas (por exemplo, com as consultas e as baixas médicas por doença). Por conseguinte, o tratamento bem-sucedido da obstipação é extremamente vantajoso.

As diretrizes acerca do tratamento da obstipação (crónica) aconselham um algoritmo de tratamento multifásico. A primeira fase consiste em alterações no estilo de vida (aumento da ingestão de líquidos, mais exercício físico, maior ingestão de fibras); a segunda fase consiste em tomar laxantes de venda livre, como bisacodilo, picossulfato de sódio e macrogol, enquanto a terceira fase implica a toma de fármacos vendidos exclusivamente mediante receita médica $[1,4]$.

Existem vários estudos que investigaram a influência do exercício físico na digestão de pacientes com obstipação e estes estudos foram agora resumidos numa meta-análise efetuada por Gao et al. [2]. Os autores identificaram 9 estudos (com um total de 680 pacientes) que examinaram o efeito do exercício físico na obstipação. Os grupos de comparação receberam acupunctura auricular, comprimidos de Maziren (medicina tradicional chinesa), formação acerca da obstipação ou nenhum tratamento em especial. As atividades desportivas foram muito variadas, mas consistiram principalmente em exercícios aeróbicos (Qigong, Baduanjin (ambos com origem na cultura asiática) ou caminhadas (fitness)); apenas um estudo investigou os efeitos do treino de força.

O treino consistiu em exercícios de 60 a 420 minutos por semana por um período entre 4 e 24 semanas. O risco relativo (proporção de risco; PR) de alívio da obstipação foi o parâmetro escolhido para avaliar os efeitos. Então o que demonstrou a análise?

Não obstante a natureza muito heterogénea dos exercícios físicos, o resultado foi bastante evidente: o alívio da obstipação melhorou em pacientes que completaram os exercícios físicos, face aos grupos de comparação. Porém, este efeito apenas foi observado após os exercícios aeróbicos (onde a PR foi 2,42 a favor dos grupos que fizeram exercícios físicos). Pelo contrário, os exercícios anaeróbicos não tiveram qualquer efeito (PR de 0,85). Se fossem combinadas as duas formas de exercício físico, então haveria ainda uma PR de 1,97. Deste modo, parece que o exercício físico foi proveitoso para aliviar a obstipação, e o exercício aeróbico durante, pelo menos, 140 minutos por semana evidenciou os melhores efeitos. Alguns dos estudos efetuados também avaliaram parâmetros como vitalidade, bem-estar e qualidade de vida e, também aqui, o exercício físico teve um efeito positivo.

Então, quais são as implicações destes resultados na prática quotidiana? O que significam, na verdade, as PRs medidas? Vamos dar um exemplo: supondo que 10 pacientes sofrem de obstipação, apenas 5 obterão alívio com o exercício físico, embora tenham feito exercício físico durante um período de entre 4 e 24 semanas (ver Figura 1, painel do lado esquerdo).

De acordo com as diretrizes atuais $[1,4]$, a primeira fase do tratamento da obstipação inclui alterações no estilo de vida (exercício físico, hidratação, fibra) e, no caso de isto não resultar, a toma de laxantes (como macrogol, bisacodilo,

Evid Self Med 2021;1:210049 | https://doi.org/10.52778/efsm.21.0049

Empresa/Correspondência: Thomas Weiser, PhD, Sabine Landes, PhD, Consumer Healthcare Medical Affairs, Sanofi-Aventis Deutschland GmbH, Industriepark Hoechst, 65026 Frankfurt am Main, Germany (thomas.weiser@sanofi.com) 


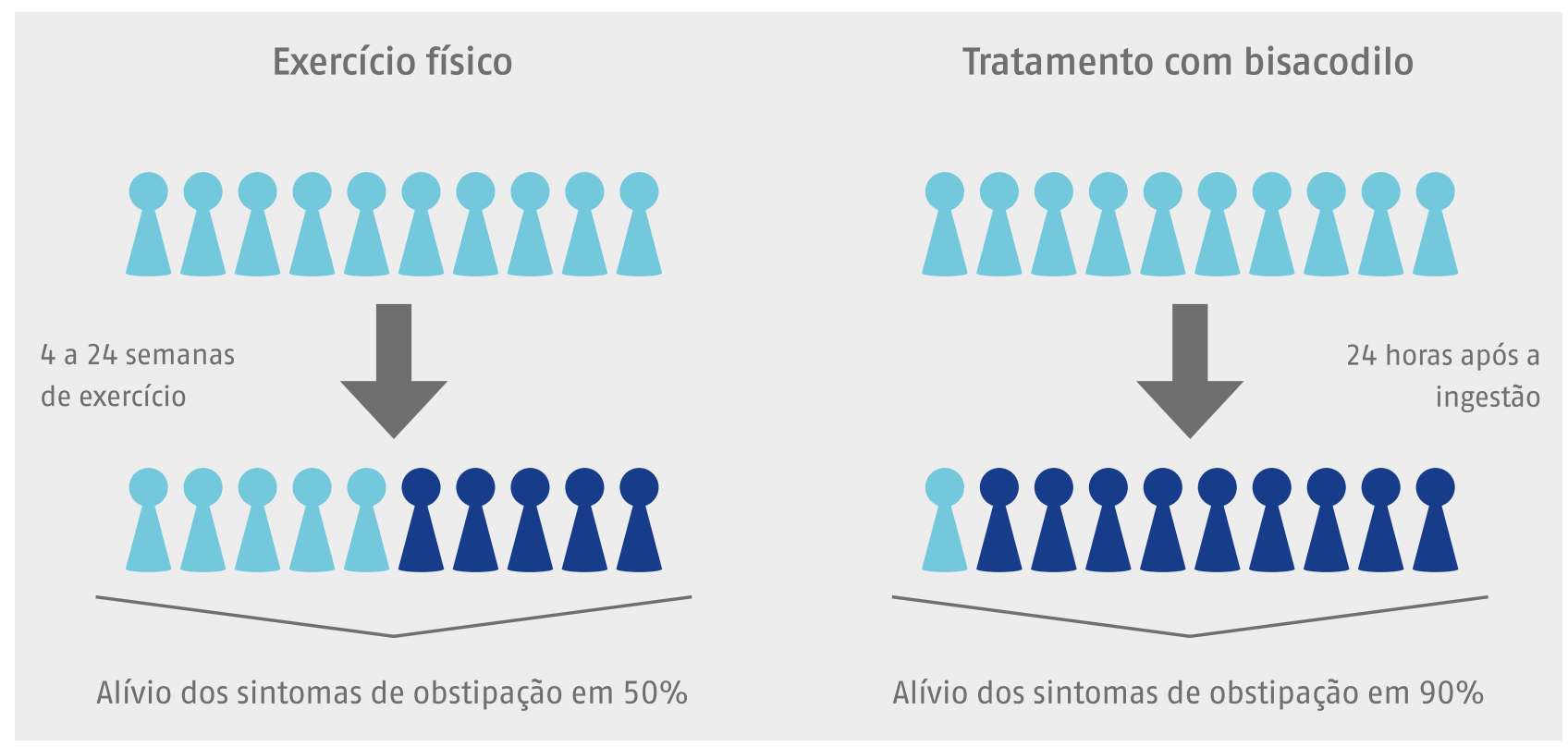

Do lado esquerdo: 0 exercício físico por um período de 4 a 24 semanas alivia a obstipação em cerca de $50 \%$ das pessoas que praticam exercício físico. Do lado direito: 0 tratamento com bisacodilo permite evacuar nas 24 horas seguintes à toma em aproximadamente $90 \%$ das pessoas tratadas.

picossulfato de sódio). Dois estudos clínicos (em que os fármacos bisacodilo ou picossulfato de sódio foram comparados com um placebo), evidenciaram que estes dois fármacos foram eficazes, seguros e bem tolerados no tratamento da obstipação. Por exemplo, cerca de $70 \%$ dos pacientes que tomaram picossulfato de sódio e $90 \%$ daqueles que usaram bisacodilo conseguiram evacuar nas primeiras 24 horas após a ingestão [3, 5 bem como "dados em arquivo", Sanofi Aventis Deutschland GmbH; Figura 1, painel do lado direito], e o alívio dos sintomas de obstipação foi registado ao longo de todo o período do estudo ( 4 semanas em cada um dos casos).

Conclusões: De acordo com Gao [2], o exercício físico durante várias semanas tem um efeito positivo na obstipação em cerca de metade das pessoas que praticam exercício. De uma maneira geral, os laxantes que contêm os fármacos bisacodilo ou picossulfato de sódio aliviam os sintomas logo a partir do primeiro dia após a ingestão em até $90 \%$ dos pacientes.

\section{Bibliografia}

1. Andresen et al. S2k Guideline for Chronic Constipation: Definition, Pathophysiology, Diagnosis and Therapy. Z Gastroenterol. 2013;51:651-672.

2. Gao R, Tao Y et al. Exercise Therapy in Patients with Constipation: A Systemic Review and Meta-Analysis of Randomized Controlled Trials. Scandinavian Journal of Gastroenterology. 2019;54:169-177.

3. Kamm MA, Müller-Lissner S et al. Oral Bisacodyl is Effective and Well-Tolerated in Patients with Chronic Constipation. Clinical Gastroenterology and Hepatology. 2011;9:577-83.

4. McCormick D. Managing Costs and Care for Chronic Idiopathic Constipation. The American Journal of Managed Care. 2019;25:S63S69.

5. Müller-Lissner S, Kamm MA et al. Multicenter, 4-Week, DoubleBlind, Randomized, Placebo-Controlled Trial of Sodium Picosulfate in Patients With Chronic Constipation; American Journal of Gastroenterology. 2010;105:897-903.

Conflito de interesses: T. Wieser e S. Landes são funcionários da Sanofi.

Divulgação: Publicação financiada por Sanofi Aventis Deutschland $\mathrm{GmbH}$.

Informações sobre o manuscrito

Data de entrega: 14.09 .2020

Data de aprovação: 27.12 .2020

Data de publicaç: 16.08.2021 